\title{
Physical and observational practices of unusual actions prime action verb processing
}

Beauprez Sophie-Anne, Blandin Yannick, Almecija Yves, Bidet-Ildei Christel

Université de Poitiers; Université de Tours; Centre National de la Recherche Scientifique;

Centre de Recherches sur la Cognition et l'Apprentissage (UMR 7295), Poitiers, France

Corresponding Author:

Christel Bidet-Ildei

Bâtiment A5 (CeRCA)

5 rue Théodore Lefebvre

TSA 21103 - 86073 Poitiers cedex 9

christel.bidet@univ-poitiers.fr

Phone: +33 (0)5 49454697 


\begin{abstract}
Numerous studies have highlighted a strong relationship between language and sensorimotor processes, showing, for example, that perceiving an action influences subsequent language processing. Moreover, previous studies have demonstrated that the context in which actions are perceived is crucial to enable this action-language relationship. In particular, action verb processing is facilitated when an action is perceived in its usual context (e.g., someone watering a plant) but not in an unusual context (e.g., someone watering a computer). This difference could be explained in terms of experience; because people always practice actions in accordance with the context, they have no (visual or motor) experience related to the unusual context. The aim of the present study was to test this assumption by assessing and comparing the effect of physical practice and observational learning on the action-language relationship. The results of two experiments showed a facilitation effect of both training methods. Whereas usual actions systematically prime action verb processing, the link between action and language appears for unusual actions only after training by practicing (experiment 1, physical practice) or observing (experiment 2, observational learning). Overall, these findings support the role of experience in the activation of sensorimotor representations during action verb processing.
\end{abstract}

Keywords: embodiment, physical practice, observational learning, language processing 


\section{Introduction}

According to embodied views of cognition, a relationship exists between language and the motor system regarding both their perceptive and productive sides. This idea of an actionlanguage relationship is not new and has been demonstrated in various behavioral and brain imagery studies.

At the behavioral level, the links between action and language have been assessed with regard to different aspects of language. For example, the production of syllables (Gentilucci, 2003; Gentilucci, Santunione, Roy, \& Stefanini, 2004), words (Bernardis \& Gentilucci, 2006) and sentences (Glenberg \& Kaschak, 2002; Setola \& Reilly, 2005) has been used to explore this action-language relationship. One example is the study by Setola and Reilly (2005), who examined the influence of action execution on verb recognition. The participants had to perform actions while performing a lexical decision task involving verbs and pseudoverbs. An interesting pattern of results was obtained in which the pseudowords were more likely to be misjudged as real words when they were cued by a "corresponding" action. For example, the pseudoword "presh" was judged as a real word when the participants simultaneously performed a pressing gesture with the hand.

Interestingly, the majority of studies have focused on how action execution influences language or how it is influenced by language. However, similar results have been obtained with action perception instead of action execution. For example, Gentilucci and his colleagues (2004) found that syllabic production was influenced by action visual perception as well as by action execution. They analyzed the kinematics of the lips and voice spectrum when participants performed or visually perceived arm actions (bringing a fruit to the mouth) while pronouncing the syllable "BA". The results showed an influence of the (executed or perceived) action on the syllable pronunciation with an increase in the second formant of the 
vowel, while a meaningless gesture led to no change. Recent studies (Beauprez \& Bidet-Ildei, 2017; Liepelt, Dolk, \& Prinz, 2012) have confirmed the influence of action perception on language processing. For example, participants in the study by Beauprez and Bidet-Ildei (2017) performed a lexical decision task on action verbs faster after being primed by a congruent action (observation of a point-light display performing the action of the verbs) than by an incongruent action (observation of another action).

It has been proposed that this influence of execution/perception of actions on language processing is sustained by the existence of a particular class of neurons in the cerebral cortex: the mirror neurons. Mirror neurons have the unique property of firing during both the execution and observation of an action (Rizzolatti \& Craighero, 2004). More generally, the mirror neuron system is considered an integral part of action-comprehension circuits. Indeed, it is suggested that the functional role of the mirror neuron system is to represent actions (Jeannerod, 2001). Thus, understanding an action produced by others relies on "resonance" processing: when observing an action, the same neural circuits are activated that would be active when one performs the action. A similar explanation could account for language comprehension and, in particular, for action language processing. Thus, when a person reads an action verb, it activates the sensorimotor representations linked to this action, similar to what occurs when the action is performed. In this sense, word meanings are built from sensorimotor experiences and are themselves the reactivations of experiences (Barsalou, 1999). In other words, the understanding of word meaning is embodied (Feldman \& Narayanan, 2004; Glenberg \& Robertson, 2000).

Neuroimaging studies support this idea by demonstrating that the system involved in language shares a neural substrate with the control of actions (Hauk, Johnsrude, \& Pulvermüller, 2004; Tettamanti et al., 2005). For example, Hauk et al. (2004) observed an overlap between the brain activations induced by the reading of verbs describing actions of 
the legs ("kick"), of the face ("lick") and of the hand ("pick") and brain activations induced by the actual execution of actions linked to these body parts.

However, studies have demonstrated that the activation of the mirror neuron system and, more generally, this resonance phenomenon depend on certain characteristics of the action perceived. For example, several studies have reported that their participants showed less or no mirror neuron system activation when the observed action was produced by a robot rather than by a human being (see Liepelt \& Brass, 2010; Matsuda, Hiraki, \& Ishiguro, 2015 for examples), when the action was performed by someone from the outgroup rather than the ingroup (Gutsell \& Inzlicht, 2010), and when the action observed was impossible rather than possible (Liepelt, Von Cramon, \& Brass, 2008; Stevens, Fonlupt, Shiffrar, \& Decety, 2000). The mirror neuron system has also been shown to be modulated by contextual differences between stimuli (Iacoboni et al., 2005). For example, in the study by Iacoboni and his colleagues (2005), greater activations of the mirror neuron networks were recorded for actions embedded in contexts (e.g., the motion of grasping a cup presented with other objects used during a tea break) compared with actions alone (e.g., the motion of grasping a cup presented without other objects).

Following this idea, if the action-language relationship is sustained by the mirror neuron system, we could assume that this relationship is also modulated by the characteristics of the action observed. This idea has been supported by recent studies (Beauprez, Toussaint, \& Bidet-Ildei, 2018; Beauprez, Laroche, Perret, \& Bidet-Ildei, 2019) focusing on the context of the action. The context refers to the environment in which an action is performed and the agent performing the action. Indeed, actions are not perceived in isolation but are embedded with objects, actors and the relationships among them. In these studies, the participants saw a prime picture depicting an action performed in a usual context (i.e., the action corresponds to the context, such as "someone watering a plant") or an unusual context (i.e., the action does 
not fit the context, such as "someone watering a computer"). Then, the participants had to perform a semantic decision task on an action verb that could be congruent (to water) or incongruent (to pay) with the previously seen prime. The results revealed that the participants answered faster when the prime and the verb were congruent, but only when the context was usual. In the unusual context, no differences between the congruent and incongruent verbs were reported. Obviously, the context is related to the motor experience of the agent. The authors proposed that these results should be interpreted as showing that context could play a role in the action-language relationship.

Studies have demonstrated an influence of motor experience on motor resonance. These studies suggest that only motor acts that are present in the motor repertoire of a person are effective in activating the mirror system (Buccino et al., 2004), or, at least, that the closer an action is to the motor repertoire, the stronger the motor resonance is (Calvo-Merino, Glaser, Grèzes, Passingham, \& Haggard, 2005). Moreover, action word processing is influenced by the motor repertoire (Lyons et al., 2010; Tomasino, Guatto, Rumiati, \& Fabbro, 2012). For example, Lyons and his colleagues (2010) demonstrated that hockey players showed greater activation of the motor cortex when listening to action sentences involving words related to hockey than did people with no hockey-related experience.

The results of Beauprez, Toussaint and Bidet-Ildei (2018) and of Beauprez and her colleagues (2019) could be explained by the same idea. Indeed, in the everyday life, people rarely (or never) produce actions in an unusual context. In contrast, all our motor experience was embedded in usual contexts. Thus, it is probable that the context(s) was (were) specifically associated with each known action in our motor repertoire. Consequently, when the participants observed actions in the usual context (close to their motor experience), their sensorimotor representations were normally activated, whereas when the participants perceived actions in the unusual context (far from their motor experience), their sensorimotor 
representations were less or not activated, leading to the absence of action-language relationships in this situation.

One way to assess whether the motor experience is involved in these effects is to use motor training paradigms. If the absence of a link between action perception and action verb processing (in the unusual context) is related to the absence of motor experience, then providing this motor experience to our participants should correct this. This was the aim of the first experiment of this paper. In this experiment, participants practiced several unusual actions to compare the influence of perceiving practiced unusual actions and unpracticed unusual actions on action verb judgments.

However, physical practice is not the only way to acquire sensorimotor representations of actions and to learn new skills. Indeed, neuroimaging studies have reported that a set of common neural structures are activated during both action production and action observation (e.g., Decety et al., 1997; Grèzes \& Decety, 2001). This is consistent with results indicating that variables that affect learning through physical practice affect observational learning in a similar way (Blandin, Proteau, \& Alain, 1994; Badets \& Blandin, 2004, 2010; Shea, Wulf, Whitacre, \& Park, 2001; Wright, Li, \& Coady, 1997). Moreover, motor skills can be acquired by observation without physical practice. For example, Cross and his colleagues (2009) trained participants for 5 days on dance sequences and recorded brain imaging data (fMRI) prior to and immediately after a physical or an observational training. The imaging data showed similar brain activity for observational and physical learning. These results demonstrate that the emergence of motor resonance is possible without physical practice and that it shows similarities for physical and observational learning (Boutin et al., 2010).

Therefore, the aim of the second experiment was to assess whether observation of usual actions can enable the emergence of the link between action and language. In this experiment, 
participants had to observe several unusual actions performed by a model before completing an action verb judgment task

\section{Experiment 1: Physical practice}

The aim of the first experiment was to assess whether the practice of unusual actions can enable the link between action perception and action verb processing. As in Beauprez et al. (2018) and Beauprez et al. (2019), we hypothesized that no action-language relationship should be obtained after the participants were primed by unpracticed unusual actions because these actions are not represented with these contexts in the motor repertoire of the participants. However, practiced unusual actions should enable the action-language relationship to appear because known actions would be associated with a new context of use in the motor repertoire of participants thanks to the motor training. In other words, action verb judgment should be facilitated only for usual actions and practiced unusual actions, not for unpracticed unusual actions.

\section{Method}

\subsection{Participants}

Twenty French university students $(\mathrm{M}=21$-year-old, $\mathrm{SD}=2.61 ; 11$ male, 19 right-handed $)$ participated in this experiment. All participants had normal or corrected-to-normal vision and no history of motor, perceptual or neurological disorders. Moreover, all participants provided written informed consent prior to their inclusion in the experiment. They were unaware of the purpose of the study.

\subsection{Prime and Stimuli}

\section{Prime Pictures:}


One hundred different pictures depicting an actor performing an action were used.

Fifty different actions were represented in these pictures. Each action was presented in both a usual and an unusual context, leading to a set of 100 pictures (see appendix 1 for examples). Thus, the same action (e.g., "to paste") had a usual picture (someone pasting paper) and an unusual picture (someone pasting a zucchini).

\section{Stimuli:}

One hundred verbs were used. Half of them were "action verbs" corresponding to the 50 actions represented in the pictures, and the other half were "non-action verbs", namely, stative verbs that do not depict a movement of the body (e.g., "to want"; see appendix 2 for the complete list of verbs). All verbs were presented in French and in the infinitive form.

\subsection{Procedure}

Two sessions were performed at an interval of $24 \mathrm{~h}$ (see fig. 1). In session 1, the participants performed an action-language priming task (approximately 20 minutes) and a learning phase (approximately 30 minutes). The next day, in session 2, the participants once again performed the action-language priming task with the same action primes and verbs and then completed a questionnaire measuring the recognition and the plausibility of the pictures presented as a prime.

SESSION 1

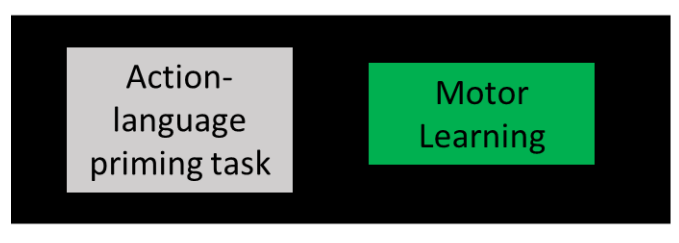

20 minutes

30 minutes

24 Hours

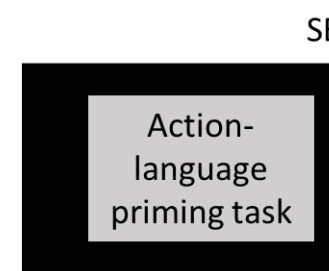

20 minutes
SESSION 2

Recognition and

Plausibility

Questionnaire

10 minutes

Figure 1. Procedure of experiment 1 (physical practice). 


\section{The action-language priming task:}

During the priming task, the participants were tested individually in a soundproof, dark room. The trials were presented on a computer screen (spatial resolution: $1280 \times 800$ pixels; temporal resolution: $60 \mathrm{~Hz}$ ) and were controlled by the software E-Prime. The experimental session included 300 trials: presentation of the 100 prime pictures (50 actions in two contexts) that could be followed by three different verbs: a congruent action verb, an incongruent action verb, or a non-action verb. Therefore, in a third of the trials, the prime was followed by a congruent action verb (for example, seeing a picture of someone skiing before seeing the word "to ski"); in another third of the trials, the prime was followed by an incongruent action verb (for example, seeing a picture of someone skiing before seeing the word "to drink"); and in the last third, the prime was followed by a non-action verb (for example, seeing a picture of someone skiing before seeing the word "to think"). This last type of trial was considered a filler, and these trials were not analyzed. They were included only to propose a task for the participants. The trial types were presented in random order.

Each trial involved the following procedure: a fixation cross appeared (500 ms), and then the prime picture (2000 ms) was presented. Following another fixation cross (500 ms), the verb stimulus appeared. It remained on the screen until the participant entered a response (see fig. 2). The participant's task was to judge, as quickly and as accurately as possible, whether the verb depicted an action. Participants answered by pressing the left button on the mouse for "yes" (for an action verb such as "to water") or the right button on the mouse for "no" (for a non-action verb such as "to want"). Thus, the participant had to perform a semantic decision task on the verb regardless of the picture previously observed. 


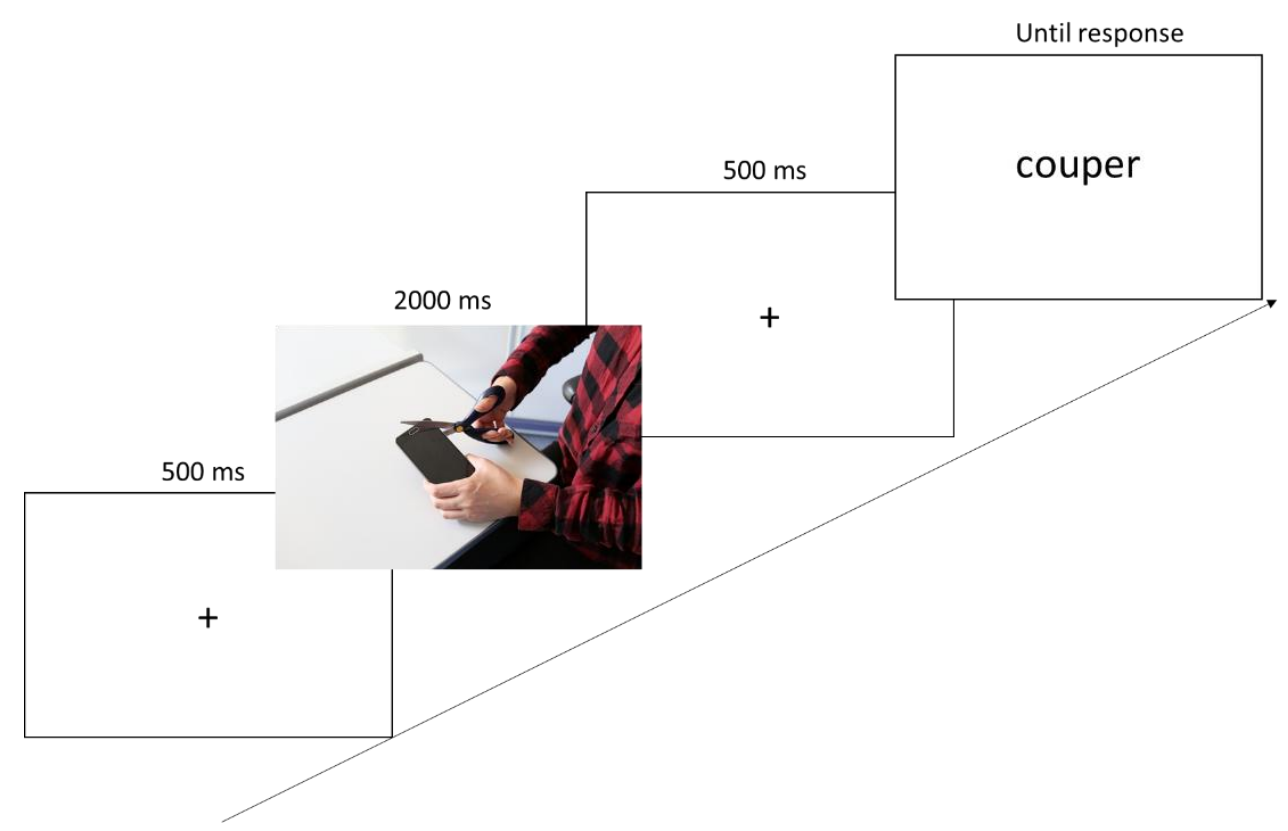

Figure 2: Procedure of the action-language priming task. The fixation cross, the prime picture and the verb stimulus (e.g., couper for "cut" in French) were centered on the screen. The arrow represents the sequence of one trial.

\section{Motor practice:}

During the learning phase, the participants had to perform certain of the unusual actions used a as prime. Among the 50 actions, 20 were practiced (see appendix 3 for the list of practiced and unpracticed unusual actions and their descriptions). During this phase, the experimenter and the participants were in a room. Each action was presented one by one, and for each action, the participants were invited to perform the proposed action four times as naturally as possible. For example, the participant saw a banana on a table, and the experimenter asked him/her to make the gesture of making a phone call with this banana. Once all the actions were performed 4 times, the participant made a second passage in which each action was performed again 4 times. 


\section{Recognition and plausibility questionnaire}

Two questionnaires were made to assess the recognition and the plausibility of each action presented. The pictures used in the priming task were presented one by one in random order, and the participants had to answer two questions. The first question asked the participants to say what action they thought was depicted. The picture obtained a score of 1 when the answer provided by the participant corresponded to the action (i.e., the participant gave the exact verb of the action or a semantically close verb) and obtained a score of 0 when the answer provided by the participant differed semantically from the one expected. A percentage of recognition per participant was calculated from these scores. The second question asked the participants to assess the plausibility of each picture using a 5-point scale ranging from "very improbable" to "very probable" (-2 to +2$)$. A score of plausibility was computed for each picture. Given our previous results about the recognition and the plausibility of unusual actions (Beauprez et al., 2018), we hypothesized that recognition would be similar for usual and unusual actions because the actions already belong to the motor repertoire. However, usual actions would be judged to be more plausible than unusual actions.

\subsection{Data analysis}

Participants' response times to the action-language priming task were analyzed after the trials, with errors and response time outliers $( \pm 2.5$ standard deviations, $7 \%$ of the data) excluded from the analysis. We used the lmer function of the lmerTest package (Kuznetsova, Brockhoff, \& Christensen, 2017) in the $\mathrm{R}$ environment ( $\mathrm{R}$ version 3.3.0, R Core Team (C) 2016) to build linear mixed-effects models. Participants and word items were specified as random-effects factors. Three fixed-effects factors were used: the type of observed picture (usual action, trained unusual action, untrained unusual action), the congruency of the verb according to the prime (congruent, incongruent) and the session $(1,2)$. Their interaction was 
also included. The $p$ values were obtained for the reported $\mathrm{F}$ values (Type III ANOVA) with the error degrees of freedom calculated based on Satterthwaite's approximation. For the questionnaires, both the recognition and the plausibility were analyzed with a non-parametric Friedman ANOVA with the type of pictures (usual, trained unusual, untrained unusual) as within factor.

\section{Results}

Analyses of response times (see fig. 3) revealed a significant interaction between the type of picture, the congruency and the session $(\mathrm{F}(2,7679)=3.803 ; p=0.02)$. Given that our hypothesis was centered on the comparison of the differences between congruent and incongruent trials for each type of picture, we proposed to complete our analysis by conducting Tuckey Post-hoc analysis.

During session 1 (i.e., before physical practice), regarding the usual pictures, the response time for congruent action verbs $(M=749.3 \mathrm{~ms}, \mathrm{SD}=133.74 \mathrm{~ms})$ was significantly shorter than for incongruent action verbs $(\mathrm{M}=808.83 \mathrm{~ms}, \mathrm{SD}=126.43 \mathrm{~ms}, p<0.01)$. However, there was no difference between congruent and incongruent action verbs for the trained unusual pictures (congruent, $\mathrm{M}=749.93 \mathrm{~ms}, \mathrm{SD}=101.91 \mathrm{~ms}$; incongruent, $\mathrm{M}=756.43 \mathrm{~ms}, \mathrm{SD}=$ $105.4 \mathrm{~ms} ; p=0.98$ ) or for the untrained unusual pictures (congruent, $\mathrm{M}=748.99 \mathrm{~ms}, \mathrm{SD}=$ $111.1 \mathrm{~ms}$; incongruent, $\mathrm{M}=765,92 \mathrm{~ms}, \mathrm{SD}=108.43 \mathrm{~ms} ; p=0.99$ ).

During session 2 (i.e., following physical practice), there was still a significant difference between congruent action verbs $(\mathrm{M}=657.7 \mathrm{~ms}, \mathrm{SD}=138.17 \mathrm{~ms})$ and incongruent action verbs $(\mathrm{M}=707.63 \mathrm{~ms}, \mathrm{SD}=122.37 \mathrm{~ms})$ for the usual pictures $(p<0.05)$. There were still no differences between congruent $(\mathrm{M}=697.12 \mathrm{~ms}, \mathrm{SD}=158.9 \mathrm{~ms})$ and incongruent action verbs $(\mathrm{M}=697.4 \mathrm{~ms}, \mathrm{SD}=137.5 \mathrm{~ms})$ for the untrained pictures $(p=1)$. However, this time, there 
was a significant difference between congruent $(\mathrm{M}=656.2 \mathrm{~ms}, \mathrm{SD}=163.3 \mathrm{~ms})$ and incongruent action verbs $(\mathrm{M}=707.9 \mathrm{~ms}, \mathrm{SD}=120.4 \mathrm{~ms})$ for the trained pictures $(p<0.05)$.

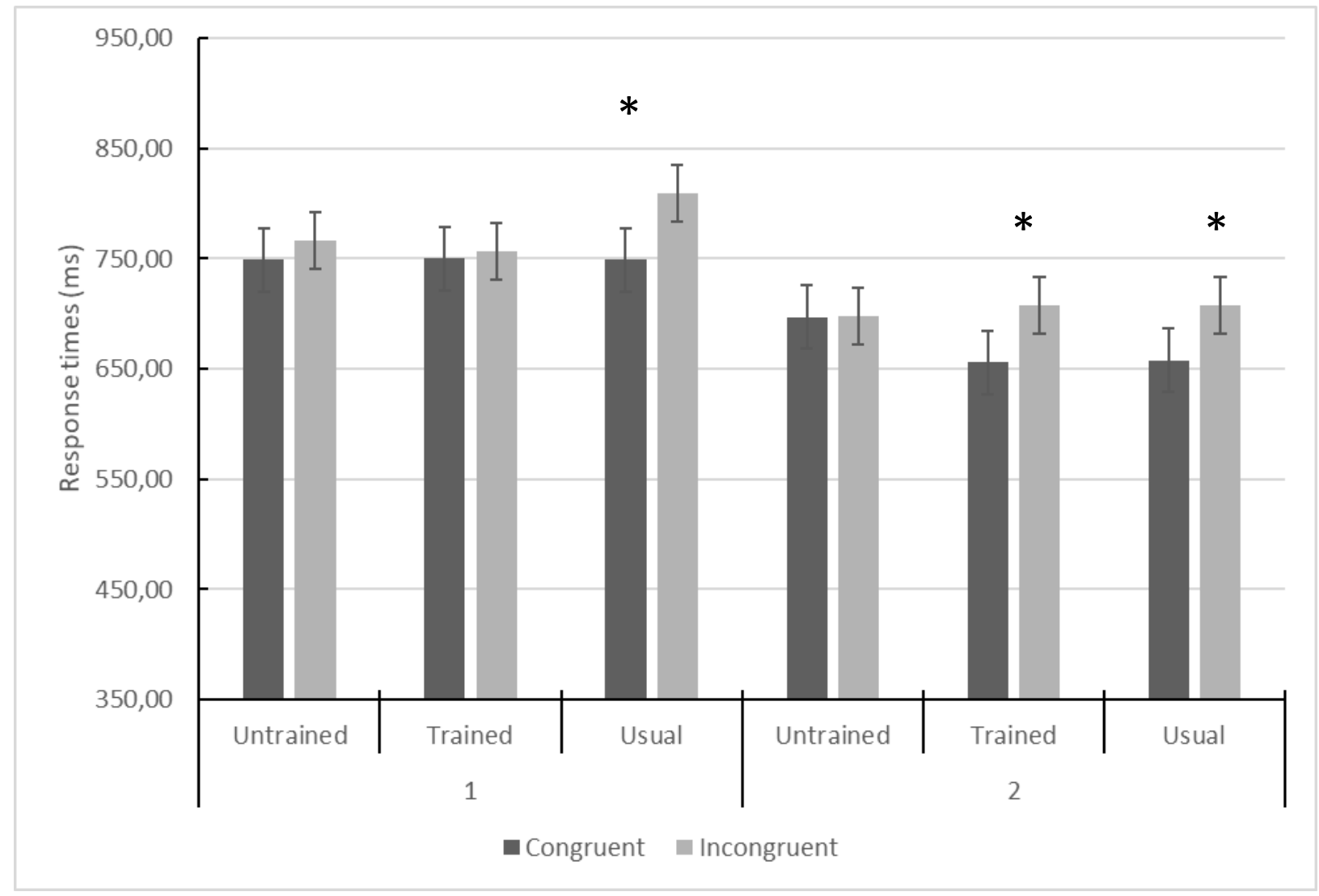

Figure 3. Mean response times (ms) according to the session $(1,2)$ and the type of action (untrained, trained, usual). The error bars indicate 95\% confidence intervals. An * indicates a significant difference $(p<0.05)$

Concerning the responses to the questionnaire, the analysis indicated that there was no difference between the recognition of usual $(\mathrm{M}=98.2 \%, \mathrm{SD}=1.9 \%)$, trained unusual $(\mathrm{M}=$ $98.8 \%, \mathrm{SD}=2.1 \%)$ and untrained unusual actions $(\mathrm{M}=97.4 \%, \mathrm{SD}=2.1 \%, \mathrm{~K}=5.8 ; p=$ 0.06). Concerning the plausibility, the analysis revealed a significant difference between the 
different types of actions $(\mathrm{K}=38.38 ; p<0.01)$. The plausibility of usual actions was higher $(\mathrm{M}=1.82, \mathrm{SD}=0.19)$ than the plausibility of trained unusual $(\mathrm{M}=-1.48, \mathrm{SD}=0.26)$ and untrained unusual actions $(\mathrm{M}=-1.21, \mathrm{SD}=0.32)$, which did not differ.

\section{Discussion}

The aim of this first experiment was to assess the role of motor experience in explaining the absence of a priming effect on a language task after perceiving an action embedded in an unusual context. As expected, when the participants had no motor experience (session 1), perceiving an unusual action led to no facilitation effect, whereas perceiving a usual action did. However, after practicing these unusual actions (session 2), the same pattern of results was found for (trained) unusual actions and usual actions. In contrast, there was still no influence of perceiving unusual actions that were not practiced.

Thus, our findings confirm that the context in which an action is embedded is essential to enabling the link between action and language (Beauprez et al., 2018; Beauprez et al., 2019), which accords with the idea that motor resonance is dependent on the action context (Amoruso, Finisguerra, \& Urgesi, 2016; Amoruso \& Urgesi, 2016).

Moreover, our findings show that short motor practice is sufficient to render operational the link between action and language when unusual actions are used. Thus, these results seem to support the assumption by Beauprez and her colleagues (2018). The link between action and language is dependent on the motor experience a person has with the action perceived. More specifically, it seems that the absence of an effect in session 1 (before motor practice) when the pictures represented unusual actions could be due to less motor resonance related to the fact that unusual contexts were not part of the perceiver's motor repertoire. However, in 
session 2, providing this motor experience to the participants enabled them to resonate with unusual actions.

Importantly, according to our hypothesis, both usual and unusual actions (practiced and unpracticed) were very well recognized, suggesting that the link between action and language is independent of the explicit recognition of actions (see Beauprez et al., 2018 and Beauprez \& Bidet-Ildei, 2018 for similar results). Concerning the plausibility, regardless of practice, unusual actions are judged less probable than usual actions, suggesting that brief motor practice is not sufficient to modify the explicit judgment of plausibility.

Globally, this first experiment shows that motor experience is a key to enable the existence of the action-language relationship independently of the explicit recognition and the plausibility judgment of actions. However, we could ask whether this motor experience is essential or whether visual experience could be sufficient to compensate for the absence of motor experience.

\section{Experiment 2: Observational learning}

As mentioned earlier, human beings have the powerful capacity to improve by observation alone without concurrent practice (Mattar \& Gribble, 2005; Torriero, Oliveri, Koch, Caltagirone, \& Petrosini, 2007). Thus, the first aim of the second study was to assess whether observational learning alone would enable the appearance of the action-language relationship for unusual actions. We hypothesized that, as in experiment 1 , before the training period, perceiving unusual actions would not influence language processing. However, after the training period, trained unusual actions as well as usual actions would facilitate the language task. It is known that the closer an action is to the motor repertoire, the more easily it resonates (Calvo-Merino et al., 2005). Following this view, previous studies have shown 
that the correspondence between the sex of the observer and the sex of the actor performing an action influences the performances during action observation (e.g., Bidet-Ildei et al., 2010). Indeed, Bidet-Ildei and collaborators (2010) showed that only the observation of a sexcongruent actor performing a running action influenced the subsequent detection of pointlight running movements embedded in a mask. Thus, in our experiment, it could be assumed that observational learning would be more efficient when participants observed someone of the same sex compared to someone of the opposite sex.

\section{Method}

\subsection{Participants}

Forty French university students participated in this experiment. Twenty were assigned to the "same sex" group $(\mathrm{M}=20$-year-old, $\mathrm{SD}=1.7 ; 12$ male, 17 right-handed $)$ and the other twenty to the "different sex" group $(\mathrm{M}=20$-year-old, $\mathrm{SD}=1.5 ; 11$ male, 19 right-handed $)$. All participants had normal or corrected-to-normal vision and no history of motor, perceptual or neurological disorders. Moreover, all participants provided written informed consent prior to their inclusion in the experiment. They were unaware of the purpose of the study.

\subsection{Prime and Stimuli}

The prime and stimuli were the same as those used in the physical practice experiment.

\subsection{Procedure}

As in the physical practice experiment, there were two sessions in this experiment (see fig. 4). During all sessions, the participants were in a quiet room with a computer screen (spatial resolution: $1280 \times 800$ pixels; temporal resolution: $60 \mathrm{~Hz}$ ). In session 1, the participants had to perform an action-language priming task (identical to the one used in the motor practice 
experiment) in which a usual or an unusual picture was followed by a verb. For each verb, the participants had to judge whether it was an action verb. Just after the priming task, the participants were visually exposed to a video representing an actor who was practicing 20 unusual actions (these actions were identical to those practiced in the motor practice experiment). In the video, each action was practiced four times in a row, and each participant saw the video two times, so each participant saw each unusual action 8 times. To assess the role of sex congruency between the actor presented in the video and the participant, two videos were used. One video presented a man, and the other presented a woman. Videos were captured with a first-person perspective to facilitate identification. Following a counterbalanced order, each participant was associated with one of these videos. More precisely, 10 women and 13 men saw a video in which a man performed the unusual actions, and 10 women and 12 men saw a video in which a woman performed the unusual actions. The observational learning had a duration of approximately 35 minutes. Twenty-four hours later, the participants started session 2. Once again, they performed the priming task and then had to complete a questionnaire measuring the recognition and the plausibility of each picture used as a prime in the priming task. This questionnaire was identical to the one used in the motor practice task.

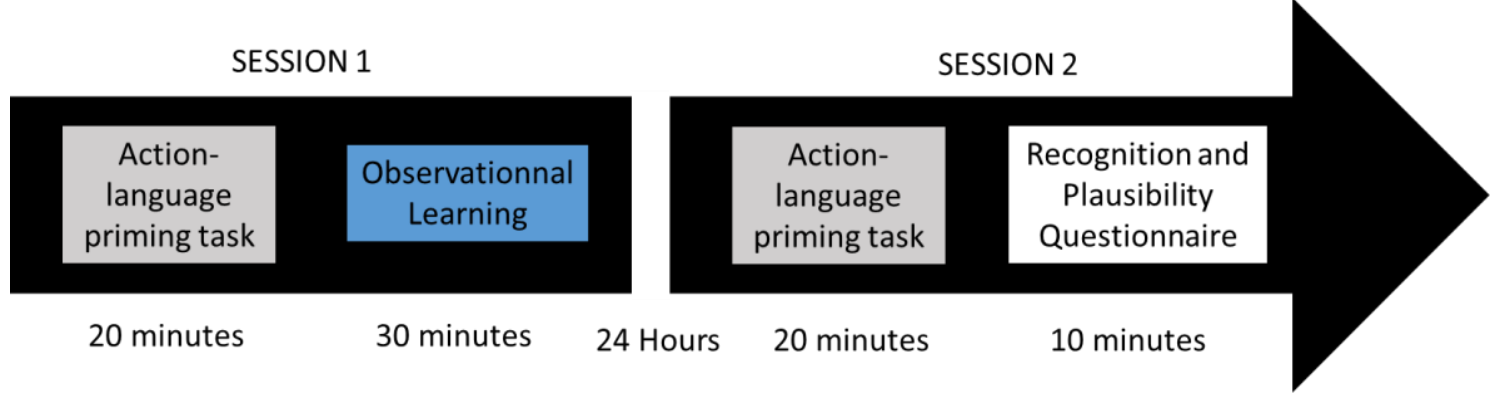

Figure 4: Procedure of experiment 2 (observational learning).

\subsection{Data Analysis}


The same analyses were performed on the participants' response times. They were analyzed after the trials, with errors and response time outliers $( \pm 2.5$ standard deviations, $10 \%$ of the data) excluded from the analyses. Four fixed-effects factors were used and included the following: the type of observed picture (usual action, trained unusual action, untrained unusual action), the congruency of the verb according to the prime (congruent, incongruent), the session $(1,2)$ and the sex congruency (same sex, different sex), and their interaction. The $p$ values were obtained for the reported F values (type III ANOVA), with the error degrees of freedom calculated based on Satterthwaite's approximation. For the questionnaires, both the recognition and the plausibility were analyzed with a non-parametric Friedman ANOVA with the type of pictures (usual, trained unusual, untrained unusual) as within factor.

\section{Results}

The analysis of the response times revealed that the interaction between the type of picture, the congruency, the session and the sex congruency was not significant $(\mathrm{F}(2,14087)=0.68 ; p$ $=0.51)$. However, the interaction between the type of picture, the congruency and the session was close to significant $(\mathrm{F}(2,14087)=2.85 ; p=0.057)$. Thus, the next analyses were performed by averaging the data of the two groups together (same sex group and different sex group were averaged).

As in Experiment 1, we performed Tuckey Post-hoc analysis to compare incongruent and congruent trials. These analyses indicated the same pattern of results as in experiment 1 (see fig. 5).

During session 1 (i.e., before the observational learning), regarding the usual pictures, shorter response times were observed for congruent action verbs $(M=675.9 \mathrm{~ms}, \mathrm{SD}=138.2 \mathrm{~ms})$ than for incongruent action verbs $(\mathrm{M}=713.1 \mathrm{~ms}, \mathrm{SD}=150.1 \mathrm{~ms})(p<0.01)$. No difference between congruent and incongruent action verbs was observed for the trained unusual pictures 
(congruent, $\mathrm{M}=687.7 \mathrm{~ms}, \mathrm{SD}=126.4 \mathrm{~ms}$; incongruent, $683.7 \mathrm{~ms}, \mathrm{SD}=106.4 \mathrm{~ms} ; p=0.97$ ) or for the untrained unusual pictures (congruent, $\mathrm{M}=673.8 \mathrm{~ms}, \mathrm{SD}=109.2 \mathrm{~ms}$; incongruent, $\mathrm{M}=685.4 \mathrm{~ms}, \mathrm{SD}=128.4 \mathrm{~ms} ; p=0.99)$.

During session 2 (i.e., after the observational learning), the difference between congruent action verbs $(\mathrm{M}=564.4 \mathrm{~ms}, \mathrm{SD}=129.2 \mathrm{~ms})$ and incongruent action verbs $(\mathrm{M}=612.97 \mathrm{~ms}$, $\mathrm{SD}=126.53 \mathrm{~ms})$ for the usual pictures $(p<0.001)$ was still observed. For the untrained unusual pictures, there was still no difference between congruent $(\mathrm{M}=583.13 \mathrm{~ms}, \mathrm{SD}=$ $127.28 \mathrm{~ms})$ and incongruent action verbs $(\mathrm{M}=596.92 \mathrm{~ms}, \mathrm{SD}=114.03 \mathrm{~ms} ; p=0.92)$. Once again, there was a significant difference between congruent $(\mathrm{M}=565.95 \mathrm{~ms}, \mathrm{SD}=121.94$ $\mathrm{ms})$ and incongruent action verbs $(\mathrm{M}=601.42 \mathrm{~ms}, \mathrm{SD}=121.5 \mathrm{~ms})$ for the trained unusual pictures $(p<0.01)$.

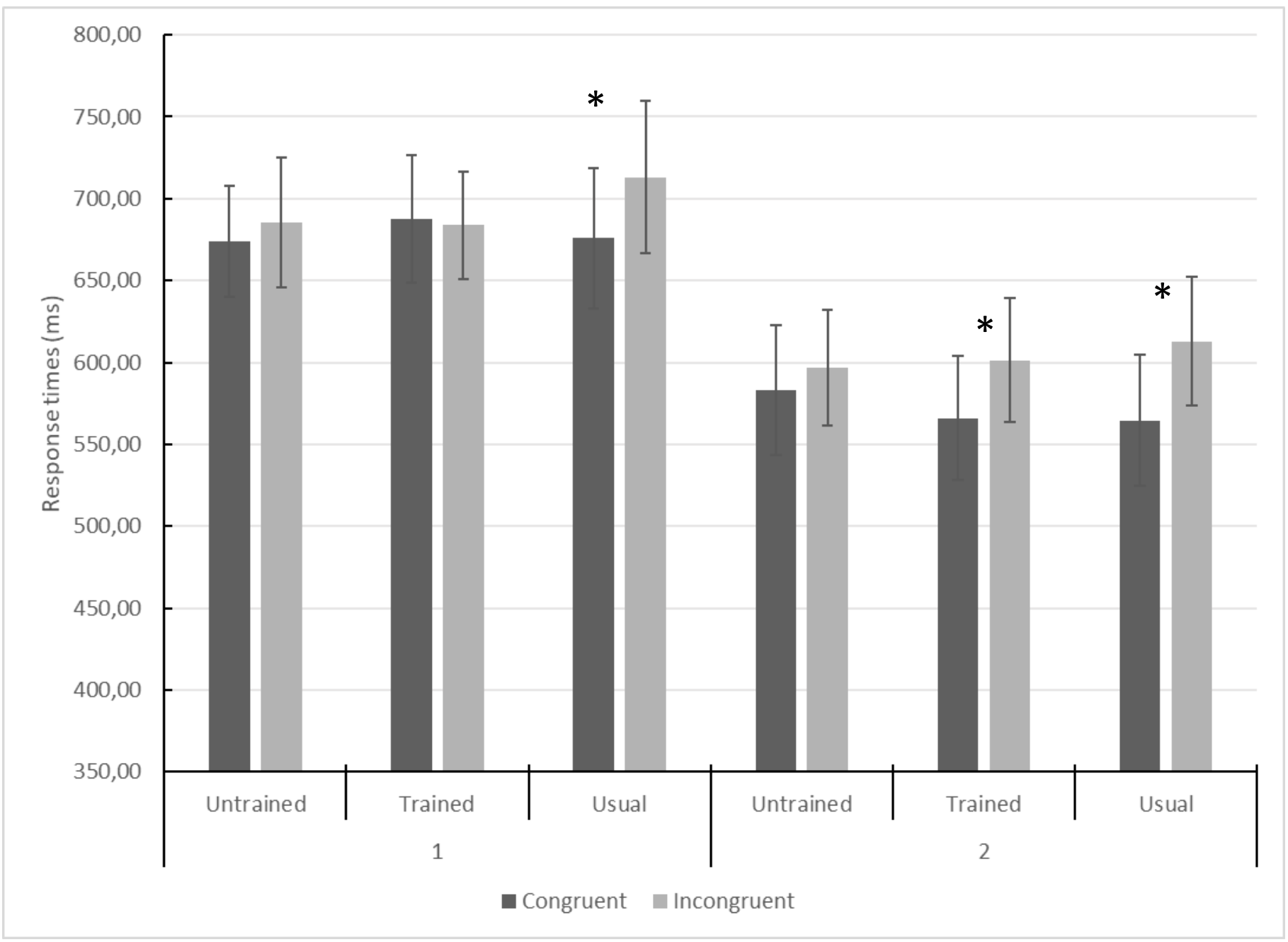


Figure 5. Mean response times (ms) according to the session $(1,2)$ and the type of action (untrained, trained, usual). The error bars indicate $95 \%$ confidence intervals. An * indicates a significant difference $(\mathrm{p}<0.05)$

Concerning the responses of the questionnaire, the analysis indicated that there was no difference between the recognition of usual $(\mathrm{M}=97.7 \%, \mathrm{SD}=2.1 \%)$, trained unusual $(\mathrm{M}=$ $97.6 \%, \mathrm{SD}=3.9 \%)$ and untrained unusual actions $(\mathrm{M}=96.7 \%, \mathrm{SD}=3.5 \%, \mathrm{~K}=2.81 ; p=$ 0.24). Concerning the plausibility, the analysis revealed a significant difference between the different types of actions $(\mathrm{K}=58.42 ; p<0.001)$. The plausibility of usual actions was higher $(\mathrm{M}=1.68, \mathrm{SD}=0.54)$ than the plausibility of trained unusual $(\mathrm{M}=-1.34, \mathrm{SD}=0.69)$ and untrained unusual actions $(\mathrm{M}=-1.17, \mathrm{SD}=0.63)$, which did not differ.

\section{Motor practice and observational learning comparison}

To compare the effect of motor practice and observational learning, we calculated the difference between the incongruent and congruent trials for each condition. We considered this difference to reflect the existence of the action-language relationship because it demonstrates that the semantic decision task is influenced by the prior perception of the action corresponding to the verb of this task.

To compare the mean differences in the condition, ANOVA was used, including the type of training (motor practice, observational learning) as a between-subject factor and the type of picture (trained, untrained, usual) and the session $(1,2)$ as within-subject factors. The significance level was fixed at $p<0.05$.

The analysis of the results confirmed an effect of the type of picture $(\mathrm{F}(2,59)=19.75 ; p<$ 0.001 , part- $\left.\eta_{\mathrm{p}}{ }^{2}=0.25\right)$ and that this effect was modulated by the session $(\mathrm{F}(2,118)=5.47 ; p<$ 
0.01 , part- $\left.\eta_{\mathrm{p}}{ }^{2}=0.08\right)$. However, there was no interaction between the type of training, the type of picture and the session $\left(\mathrm{F}(2,118)=0.43 ; p=0.65\right.$, part- $\left.\eta_{\mathrm{p}}{ }^{2}=0.007\right)$. The absence of the type of training effect indicated that the facilitation effect provided by practice was similar (the difference between incongruent and congruent conditions in session 2) for physical (51.7 ms) and observational practices (35.4 ms).

\section{Discussion}

The aim of the second experiment was to assess whether observation alone could allow us to create the necessary experience for participants to resonate when perceiving an action in an unusual context. An interaction, close to significance, was obtained between the session, the type of picture and the congruency, indicating that the results of interest were similar to those obtained in experiment 1 . During session 1, perceiving an unusual action led to no facilitation effect, whereas perceiving a usual action did. After observational learning, a facilitation effect was obtained for both (trained) unusual actions and usual actions, whereas there was still no influence of unusual actions that were not practiced. Futures studies should be carried out to confirm those results. Anyway, those results accords with the idea that experience is essential for the link between action and language. Interestingly, these findings also showed that visual exposition alone is efficient, which accords with the idea that the action-language relationship is related to the activation of sensorimotor representations (Bidet-Ildei \& Toussaint, 2015). Moreover, as was the case for experiment 1, both usual and unusual actions (practiced and unpracticed) were very well recognized, and unusual actions were judged less probable than usual actions.

It is worth noting that there was no influence of the sex of the actor. We assumed that the learning would be facilitated when the sex of the observer and the observed was the same, 
but this was not the case. This lack of effect could be related to the type of action used in the experiment. The actions used were essentially related to fine motor competencies (e.g., staple, sign, screw), whereas previous studies that demonstrated a role of sex used global motor competencies (e.g., locomotion, Bidet-Ildei, Chauvin, \& Coello, 2010). Therefore, it is probable that fine motor skills are not sufficiently distinguishable between males and females to show an effect.

\section{General Discussion}

The present study aimed to explore the role of motor or observational learning in the action-language relationship. In these experiments, we presented to the participants a picture depicting someone performing an action in a usual or unusual context before asking them to complete a language decision semantic task. The results showed that the presentation of the pictures facilitated the processing of the verb related to the action presented, but only when the context of this action was usual. That is, no facilitation effect was obtained in association with the unusual actions. However, after training (physical practice or observational learning), pictures of unusual actions also facilitated the processing of the verbs related to these actions.

These findings confirm that the context of an action is essential in the action-language relationship (Beauprez et al, 2018; Beauprez et al., 2019) and show for the first time that the absence of effect when the context is unusual is directly related to the absence of motor and/or visual experience. Thus, the findings confirm that the action-language relationship is related to the activation of specific sensorimotor representations (Bidet-Ildei \& Toussaint, 2015) that are directly related to the strength of motor experience (Lyons et al., 2010; Tomasino et al., 2012). However, it is interesting to note that our results also demonstrated that motor practice 
is not essential because the visual practice of action was sufficient to enable the effect. Therefore, our results are in agreement with numerous experiments indicating that observing someone enhances motor learning, whether for laboratory tasks (e.g., Boutin et al., 2010) or for ecological situations related to sports (e.g., D’Innocenzo, Gonzalez, Williams, \& Bishop, 2016) or rehabilitation (e.g., Park, Song, \& Kim, 2014). Thus, the results of the present study agree with behavioral and brain activation studies that have demonstrated that observational and physical learning rely on similar cognitive processes and neuronal substrates (Blandin et al., 1999; Boutin et al., 2010; Cross et al., 2009). Our results also agree with a recent study demonstrating that visual experience enables the creation and/or reinforcement of the link between action and language (Beauprez, Bidet-Ildei, \& Hiraki, 2019). In this study, a visual familiarization of only 10 minutes with an unusual agent (i.e., a robot) was sufficient for the action-language link to appear.

For the action-language link investigated in the present study, the physical and observational learning methods appeared to be equally efficient; however, we could have expected that physical practice would be more efficient than observational learning. Indeed, our findings seem to be in contradiction with other studies that demonstrated that physical practice can be more efficient than observational learning with regard to motor resonance (Calvo-Merino, Grèzes, Glaser, Passingham, \& Haggard, 2006). Calvo-Merino and her colleagues (2006) recorded the cerebral activity of male and female classical ballet dancers. In classical ballet, certain moves are sex specific. Thus, even if all dancers have equal visual experience with all moves, they have motor experience only with the moves specific to their sex. The results of their experiment demonstrated greater activation in the premotor cortex when dancers perceived moves belonging to their motor repertoire in comparison with moves of the opposite sex. However, there is a crucial difference between the results of CalvoMerino et al. (2006) and ours. They compared actions belonging to the motor repertoire with 
actions that do not belong to it. In contrast, in our study, all actions presented belonged to the motor repertoire of the participants. For example, even if our participants did not have the experience of biting a hammer, the action of "biting" was already present in the motor repertoire of the participants. Therefore, to be able to resonate when perceiving an action, perhaps visual experience is not sufficient for movements that do not belong to the motor repertoire, but it seems that it is sufficient to enrich existing motor representations with a new context of use.

Another important implication of our study is that the link between action and language has a certain flexibility. Indeed, short motor or visual experience directly affects the link between action and language, suggesting that visual or motor experience enables us to extend our motor resonance capacities (Beauprez et al., 2019; Buccino et al., 2004). Moreover, our data support the idea that the action-language relationship relies to associative learning (Pulvermüller, 1996, 1999). Actually, the association theory suggests that the link is created progressively by repetitively associating words and actions (Pulvermüller, 2005) based on the Hebbian theory, which holds that the simultaneous activation of separate cells can generate a functional unity (Hebb, 1949). In other words, when two neurons are regularly activated simultaneously, they reinforce their connection. That is, the activation of one of these two neurons automatically leads to the activation of the other. In his model, Pulvermüller (1996, 1999) proposed that words frequently encountered in context involving physical activity (e.g., action verbs) are coded by networks distributed in language and motor areas. This idea is supported by studies demonstrating that association learning changes the brain's motor response to stimulus, particularly when we learn to associate novel word-forms with motor action (see Fargier et al., 2012 for an example). In their study, participants were trained to associate novel verbal stimuli with videos of object-oriented hand and arm movements. At the end of training, strong language-induced $\mu$ suppression (considered an 
indicator of motor activity) was observed. In agreement with this finding, our behavioral data revealed that the link between performed actions or observed actions and action verbs is rapidly established with training. In fact, through repeated associations between the verb and an unusual context (with motor or observation learning), our study showed that it is possible to render operational the link between action and language in only one session of half an hour ( 8 trials by action).

Conversely, motor practice and observational learning had no effect on the explicit recognition of action and the judgment of plausibility. In both experiments, independent of the practice, the recognition of usual and unusual actions was very high, and the plausibility was weaker for unusual actions than usual actions. This finding confirms that the link between action and language is based on an implicit (Beauprez et al., 2018; Beauprez \& Bidet-Ildei, 2018; Willems et al., 2010) use of motor representations and suggests that practicing or observing actions for half an hour is not sufficient to modify the explicit judgments of participants. However, it is also possible that the questionnaires used were not sufficiently sensitive to highlight a potential practice effect on the explicit measures.

Finally, our study may have a direct impact on the rehabilitation of language disorders with action observation. Several works have shown that action observation can be used to rehabilitate patients suffering from nonfluent aphasia (see Marangolo \& Caltagirone, 2014 for a review). When action observation is associated with intensive language training, patients with nonfluent aphasia present a better recovery of language function than when language training is associated with the observation of meaningless movements (Marangolo et al., 2010). Interestingly, this effect is dependent on the action presented. Indeed, only the observation of human actions (compared with animal or mechanical actions) can positively improve performance in language (Marangolo et al., 2012). With our study, we underline that learning or relearning verbs is optimized when the verbs are associated with their usual 
context of practice. Therefore, the association of the action with the usual context can act as a catalyzer to emphasize the link between action and language and optimize the rehabilitation of patients suffering from language disorders. Future studies should specifically test this assumption with patients.

Ethical approval: The experiments were conducted in accordance with the ethical standards of the institution and with the 1964 Helsinki Declaration.

Acknowledgements: Anne-Claire Boisson, Erwan Metais and Mathilde Jousson participated in the data collection.

\section{References}

Amoruso, L., Finisguerra, A., \& Urgesi, C. (2016). Tracking the Time Course of Top-Down Contextual Effects on Motor Responses during Action Comprehension. Journal of Neuroscience, 36(46), 11590-11600. https://doi.org/10.1523/JNEUROSCI.434015.2016

Amoruso, L., \& Urgesi, C. (2016). Contextual modulation of motor resonance during the observation of everyday actions. NeuroImage, 134, 74-84. https://doi.org/10.1016/j.neuroimage.2016.03.060.

Badets, A., \& Blandin, Y. (2004). The role of knowledge of results frequency in learning through observation. Journal of Motor Behavior, 36(1), 62-70. https://doi.org/10.3200/JMBR.36.1.62-70

Badets, A., \& Blandin, Y. (2010). Feedback schedules for motor-skill learning: the similarities and differences between physical and observational practice. Journal of Motor Behavior, 42(4), 257-268. https://doi.org/10.1080/00222895.2010.497512

Barsalou, L. W. (1999). Perceptual symbol systems. The Behavioral and Brain Sciences, 22(4), 577-609. https://doi.org/10.1017/S0140525X99002149 
Beauprez, S.-A., \& Bidet-Ildei, C. (2017). Perceiving a Biological Human Movement Facilitates Action Verb Processing. Current Psychology, 1-5. https://doi.org/10.1007/s12144-017-9694-5

Beauprez, S. A., \& Bidet-Ildei, C. (2018). The kinematics, not the orientation, of an action influences language processing. Journal of Experimental Psychology. Human Perception and Performance. https://doi.org/10.1037/xhp0000568

Beauprez, S.-A., Bidet-Ildei, C., \& Hiraki, K. (2019). Does watching Han Solo or C-3PO similarly influence our language processing? Psychological Research. https://doi.org/10.1007/s00426-019-01169-3

Beauprez, S.-A., Laroche, B., Perret, C., \& Bidet-Ildei, C. (2019). How action context modulates the action-lnguage relationship: a topograpic ERP analysis. Brain Topography.

Beauprez, S.-A., Toussaint, L., \& Bidet-Ildei, C. (2018). When context modulates the influence of action observation on language processing. PLOS ONE, 13(8), 1-12. https://doi.org/10.1371/journal.pone.0201966

Bernardis, P., \& Gentilucci, M. (2006). Speech and gesture share the same communication system. $\quad$ Neuropsychologia, $\quad 44(2), \quad$ 178-190. https://doi.org/10.1016/j.neuropsychologia.2005.05.007

Bidet-Ildei, C., Chauvin, A., \& Coello, Y. (2010). Observing or producing a motor action improves later perception of biological motion: evidence for a sex effect. Acta Psychologica, 134(2), 215-224. https://doi.org/10.1016/j.actpsy.2010.02.002

Bidet-Ildei, C., \& Toussaint, L. (2015). Are judgments for action verbs and point-light human actions equivalent? Cognitive Processing, 16(1), 57-67. https://doi.org/10.1007/s10339-014-0634-0

Blandin, Y., Lhuisset, L., \& Proteau, L. (1999). Cognitive processes underlying observational learning of motor skills. The Quarterly Journal of Experimental Psychology, 52A, 957-979. https://doi.org/10.1080/713755856

Blandin, Y., Proteau, L., \& Alain, C. (1994). On the cognitive processes underlying contextual interference and observational learning. Journal of Motor Behavior, 26, 18 26. 
Boutin, A., Fries, U., Panzer, S., Shea, C.H., \& Blandin, Y. (2010). Role of action observation and action in sequence learning and coding. Acta Psychologica, 135, 240-251.

Buccino, G., Lui, F., Canessa, N., Patteri, I., Lagravinese, G., Benuzzi, F., ... Rizzolatti, G. (2004). Neural circuits involved in the recognition of actions performed by nonconspecifics: an FMRI study. Journal of Cognitive Neuroscience, 16(1), 114-126. https://doi.org/10.1162/089892904322755601

Calvo-Merino, B., Glaser, D. E., Grèzes, J., Passingham, R. E., \& Haggard, P. (2005). Action observation and acquired motor skills: an FMRI study with expert dancers. Cerebral Cortex, 15(8), 1243-1249. https://doi.org/10.1093/cercor/bhi007

Calvo-Merino, B., Grèzes, J., Glaser, D. E., Passingham, R. E., \& Haggard, P. (2006). Seeing or doing? Influence of visual and motor familiarity in action observation. Current Biology, 16(19), 1905-1910. https://doi.org/10.1016/j.cub.2006.07.065

Cross, E. S., Kraemer, D. J.M., Hamilton, A. F. d. C., Kelley, W. M., \& Grafton, S. T. (2009) Sensitivity of the action observation network to physical and observational learning. Cerebral Cortex, 19(2), 315-326. https://doi.org/10.1093/cercor/bhn083

Decety, J., Grèzes, J., Costes, N., Perani, D., Jeannerod, M., Procyk, E., ... Fazio, F. (1997). Brain activity during observation of actions. Influence of action content and subject's strategy. Brain: A Journal of Neurology, 120 ( Pt 10), 1763-1777. https://doi.org/10.1093/brain/120.10.1763

D’Innocenzo, G., Gonzalez, C. C., Williams, A. M., \& Bishop, D. T. (2016). Looking to learn: The effects of visual guidance on observational learning of the golf swing. PLoS ONE, 11, e155442. https://doi.org/10.1371/journal.pone.0155442

Fargier, R., Paulignan, Y., Boulenger, V., Monaghan, P., Reboul, A., \& Nazir, T. A. (2012). Learning to associate novel words with motor actions: language-induced motor activity following short training. Cortex, 48(7), 888-899. https://doi.org/10.1016/j.cortex.2011.07.003

Feldman, J., \& Narayanan, S. (2004). Embodied meaning in a neural theory of language. Brain and Language, 89(2), 385-392. https://doi.org/10.1016/S0093-934X(03)003559

Gentilucci, M. (2003). Grasp observation influences speech production. The European Journal of Neuroscience, 17(1), 179-184. 
Gentilucci, M., Santunione, P., Roy, A. C., \& Stefanini, S. (2004). Execution and observation of bringing a fruit to the mouth affect syllable pronunciation. The European Journal of Neuroscience, 19(1), 190-202. https://doi.org/10.1111/j.1460-9568.2004.03104.x

Glenberg, A. M., \& Kaschak, M. P. (2002). Grounding language in action. Psychonomic bulletin \& review, 9(3), 558-565. https://doi.org/10.3758/BF03196313

Glenberg, A., \& Robertson, D. A. (2000). Symbol Grounding and Meaning: A Comparison of High-Dimensional and Embodied Theories of Meaning. Journal of Memory and Language, 43(3), 379-401. https://doi.org/10.1006/jmla.2000.2714

Grèzes, J., \& Decety, J. (2001). Functional anatomy of execution, mental simulation, observation, and verb generation of actions: a meta-analysis. Human Brain Mapping, 12(1), 1-19.

Gutsell, J., \& Inzlicht, M. (2010). Empathy constrained: Prejudice predicts reduced mental simulation of actions during observation of outgroups. Journal of Experimental Social Psychology, 46(5), 841-845. https://doi.org/10.1016/j.jesp.2010.03.011

Hauk, O., Johnsrude, I., \& Pulvermüller, F. (2004). Somatotopic representation of action words in human motor and premotor cortex. Neuron, 41(2), 301-307. https://doi.org/10.1016/S0896-6273(03)00838-9

Hebb, D. O. (1949). The Organization of Behavior. A Neuropsychological Theory. New York: Wiley.

Iacoboni, M., Molnar-Szakacs, I., Gallese, V., Buccino, G., Mazziotta, J. C., \& Rizzolatti, G. (2005). Grasping the intentions of others with one's own mirror neuron system. PLoS Biology, 3(3), e79. https://doi.org/10.1371/journal.pbio.0030079

Jeannerod, M. (2001). Neural simulation of action: a unifying mechanism for motor cognition. NeuroImage, 14(1 Pt 2), 103-109. https://doi.org/10.1006/nimg.2001.0832

Liepelt, R., \& Brass, M. (2010). Top-down modulation of motor priming by belief about animacy. Experimental Psychology, 57(3), 221-227. https://doi.org/10.1027/1618$3169 / \mathrm{a} 000028$

Liepelt, R., Dolk, T., \& Prinz, W. (2012). Bidirectional semantic interference between action and speech. Psychological Research, 76(4), 446-455. https://doi.org/10.1007/s00426011-0390-z

Liepelt, R., Von Cramon, D. Y., \& Brass, M. (2008). How do we infer others' goals from non-stereotypic actions? The outcome of context-sensitive inferential processing in 
right inferior parietal and posterior temporal cortex. NeuroImage, 43(4), 784-792. https://doi.org/10.1016/j.neuroimage.2008.08.007

Lyons, I. M., Mattarella-Micke, A., Cieslak, M., Nusbaum, H. C., Small, S. L., \& Beilock, S. L. (2010). The role of personal experience in the neural processing of action-related language. Brain and Language, 112(3), 214-222. https://doi.org/10.1016/j.bandl.2009.05.006

Marangolo, P., Bonifazi, S., Tomaiuolo, F., Craighero, L., Coccia, M., Altoe, G., ... Cantagallo, A. (2010). Improving language without words: first evidence from aphasia. Neuropsychologia, $\quad$ 48(13), 3824-3833. https://doi.org/10.1016/j.neuropsychologia.2010.09.025

Marangolo, P., \& Caltagirone, C. (2014). Options to enhance recovery from aphasia by means of non-invasive brain stimulation and action observation therapy. Expert Review of Neurotherapeutics, 14(1), 75-91. https://doi.org/10.1586/14737175.2014.864555

Marangolo, P., Cipollari, S., Fiori, V., Razzano, C., \& Caltagirone, C. (2012). Walking but Not Barking Improves Verb Recovery: Implications for Action Observation Treatment in Aphasia Rehabilitation. PLoS ONE, 7(6), 1-7. https://doi.org/10.1371/journal.pone.0038610

Matsuda, G., Hiraki, K., \& Ishiguro, H. (2015). EEG-Based Mu Rhythm Suppression to Measure the Effects of Appearance and Motion on Perceived Human Likeness of a Robot. Journal of Human-Robot Interaction, 5(1), 68-81. https://doi.org/10.5898/10.5898/JHRI.5.1.Matsuda

Mattar, A. A. G., \& Gribble, P. L. (2005). Motor learning by observing. Neuron, 46(1), 153-160. https://doi.org/10.1016/j.neuron.2005.02.009

Park, S. D., Song, H. S., \& Kim, J. Y. (2014). The effect of action observation training on knee joint function and gait ability in total knee replacement patients. Journal of Exercise Rehabilitation, 10, 168-171. https://doi.org/10.12965/jer.140112

Pulvermüller, F. (1996). Hebb's concept of cell assemblies and the psychophysiology of word processing. Psychophysiology, 33(4), 317-333.

Pulvermüller, F. (1999). Words in the brain's language. The Behavioral and Brain Sciences, 22(2), 253-279; discussion 280-336. 
Pulvermüller, F. (2005). Brain mechanisms linking language and action. Nature Reviews. Neuroscience, 6(7), 576-582. https://doi.org/10.1038/nrn1706

Rizzolatti, G., \& Craighero, L. (2004). The mirror-neuron system. Annual Review of Neuroscience, 27, 169-192. https://doi.org/10.1146/annurev.neuro.27.070203.144230

Rizzolatti, G., Fogassi, L., \& Gallese, V. (2001). Neurophysiological mechanisms underlying the understanding and imitation of action. Nature Reviews. Neuroscience, 2(9), 661-670. https://doi.org/10.1038/35090060

Scully, D. M., \& Newell, K. M. (1985). The acquisition of motor skills: toward a visual perception perspective. Journal of Human Movement Studies, 12, 169-187.

Setola, P., \& Reilly, R. G. (2005). Words in the brain's language: an experimental investigation. Brain and Language, 94(3), 251-259. https://doi.org/10.1016/j.bandl.2004.12.007

Shea, C. H., Wulf, G., Whitacre, C. A., \& Park, J. H. (2001). Surfing the implicit wave. The Quarterly Journal of Experimental Psychology. A, Human Experimental Psychology, 54(3), 841-862. https://doi.org/10.1080/713755993

Stevens, J. A., Fonlupt, P., Shiffrar, M., \& Decety, J. (2000). New aspects of motion perception: selective neural encoding of apparent human movements. Neuroreport, 11(1), 109-115.

Tettamanti, M., Buccino, G., Saccuman, M. C., Gallese, V., Danna, M., Scifo, P., ... Perani, D. (2005). Listening to action-related sentences activates fronto-parietal motor circuits. Journal of Cognitive Neuroscience, 17(2), 273-281. https://doi.org/10.1162/0898929053124965

Tomasino, B., Guatto, E., Rumiati, R. I., \& Fabbro, F. (2012). The role of volleyball expertise in motor simulation. Acta Psychologica, 139(1), 1-6. https://doi.org/10.1016/j.actpsy.2011.11.006

Torriero, S., Oliveri, M., Koch, G., Caltagirone, C., \& Petrosini, L. (2007). The what and how of observational learning. Journal of Cognitive Neuroscience, 19(10), 1656-1663. https://doi.org/10.1162/jocn.2007.19.10.1656

Willems, R. M., Toni, I., Hagoort, P., \& Casasanto, D. (2010). Neural dissociations between action verb understanding and motor imagery. Journal of Cognitive Neuroscience, 22(10), 2387-2400. https://doi.org/10.1162/jocn.2009.21386 
Wright, D. L., Li, Y., \& Coady, W. (1997). Cognitive Processes Related to Contextual Interference and Observational Learning: A Replication of Blandin, Proteau, and Alain (1994). Research Quarterly for Exercise and Sport, 68(1), 106-109. https://doi.org/10.1080/02701367.1997.10608872 


\section{Appendix 1: Example of a prime picture}

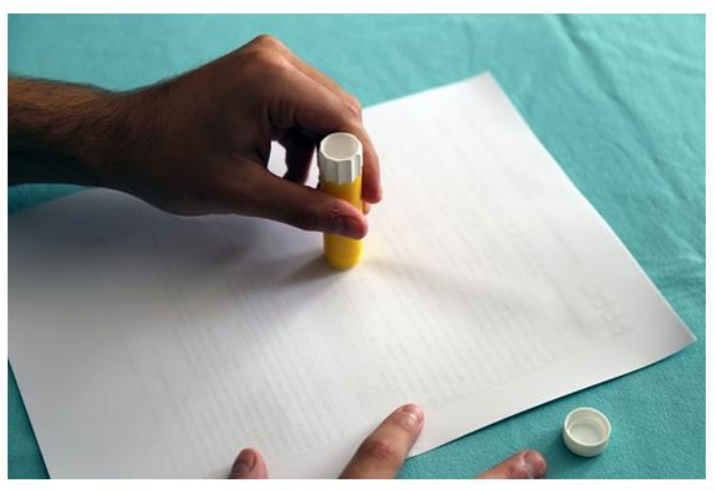

« To paste» : usual context

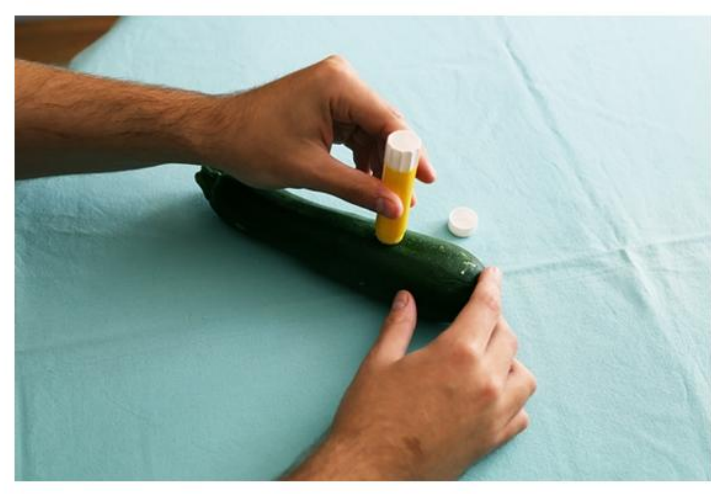

« To paste» : unusual context

Appendix 2: List of French action verbs and their English translation (in parentheses)

\begin{tabular}{|l|l|l|l|}
\hline Agrafer (to staple) & Ecouter (to listen) & Parfumer (to perfume) & Scotcher (to tape) \\
\hline Allumer (to light) & Epiler (to depilate) & Payer (to pay) & Se laver (to wash) \\
\hline Arroser (to water) & Eplucher (to peel) & Pêcher (to fish) & $\begin{array}{l}\text { Se moucher (to } \\
\text { blow) }\end{array}$ \\
\hline $\begin{array}{l}\text { Aspirer (to } \\
\text { vacuum) }\end{array}$ & $\begin{array}{l}\text { Etrangler } \\
\text { strangle) }\end{array}$ & Peigner (to comb) & Signer (to sign) \\
\hline Balayer (to sweep) & Fumer (to smoke) & Peindre (to paint) & Skier (to ski) \\
\hline Boire (to drink) & Gonfler (to inflate) & Percer (to drill) & Souffler (to blow) \\
\hline Brancher (to plug) & Jeter (to throw) & Peser (to weigh) & Téléphoner (to call) \\
\hline $\begin{array}{l}\text { Bronzer } \\
\text { sunbathe) }\end{array}$ & Jouer (to play) & $\begin{array}{l}\text { Photocopier } \\
\text { photocopy) }\end{array}$ & Tirer (to shoot) \\
\hline Brosser (to brush) & Lire (to read) & Plonger (to dive) & Tondre (to mow) \\
\hline Chanter (to sing) & Manger (to eat) & Poster (to post) & Tricoter (to knit) \\
\hline Coller (to paste) & Mixer (to blend) & Promener (to walk) & Visser (to screw) \\
\hline Coudre (to sew) & Mordre (to bite) & Ratisser (to rake) & \\
\hline Couper (to cut) & Nettoyer (to wash) & Repasser (to iron) & \\
\hline
\end{tabular}




\section{Appendix 3: Description of the unusual actions (practiced and not practiced)}

\begin{tabular}{|c|c|c|c|}
\hline \multicolumn{4}{|c|}{ Unusual actions - practiced } \\
\hline To bite & Biting a hammer & To peel & Peeling a mug \\
\hline To blow & Blowing on a bottle & To perfume & Putting perfume on an apple \\
\hline To brush & Brushing teeth with a pen & To plug & $\begin{array}{l}\text { Plugging a USB cable into a } \\
\text { grounded outlet }\end{array}$ \\
\hline To call & Calling with a banana & To rake & Raking in a living room \\
\hline To comb & Combing a kettle & To screw & Screwing a radish \\
\hline To depilate & Depilating a bottle & To sew & Sewing a sponge \\
\hline To inflate & Inflating a belly & To shoot & Shooting with a banana \\
\hline To listen & $\begin{array}{l}\text { Listening to music from an } \\
\text { apple }\end{array}$ & To sign & Signing with a carrot \\
\hline To paint & Painting a plant & To tape & Taping an apple \\
\hline To paste & Pasting a zucchini & To wash & Washing with kitchen sponge \\
\hline \multicolumn{4}{|c|}{ Unusual actions - not practiced } \\
\hline To blend & Blending furniture & To post & Posting a smartphone \\
\hline To blow & $\begin{array}{l}\text { Blowing nose in currency } \\
\text { paper }\end{array}$ & To read & Reading a book upside-down \\
\hline To cut & Cutting a smartphone & To sing & $\begin{array}{l}\text { Singing with a construction } \\
\text { mask }\end{array}$ \\
\hline To dive & Diving in the stairs & To ski & Skiing in grass \\
\hline To drill & Drilling a window & To smoke & Smoking a carrot \\
\hline To drink & Drinking detergent & To staple & Stapling paper currency \\
\hline To eat & Eating a camera & To strangle & Strangling a stuffed animal \\
\hline To fish & Fishing in a street & To sunbath & Sunbathing in a parking lot \\
\hline To iron & Ironing a CD & To sweep & Sweeping in a garden \\
\hline To knit & Knitting with candies & To throw & $\begin{array}{l}\text { Throwing paper currency in a } \\
\text { bin }\end{array}$ \\
\hline To light & Lighting pens & To vacuum & Vacuuming in a garden \\
\hline To mow & Mowing in a room & To walk & Walking a stuffed animal \\
\hline To pay & Paying with fake bills & To wash & Washing a keyboard \\
\hline $\begin{array}{l}\text { To } \\
\text { photocopy }\end{array}$ & Photocopying head & To water & Watering a laptop \\
\hline To play & $\begin{array}{l}\text { Playing monopoly with a } \\
\text { passport }\end{array}$ & To weigh & Weighing standing on hands \\
\hline
\end{tabular}

